\title{
Quantifying the growth of individual graphene layers by in situ environmental transmission electron microscopy
}

\author{
Kling, Jens; Hansen, Thomas Willum; Wagner, Jakob Birkedal
}

Published in:

Carbon

Link to article, DOI:

10.1016/j.carbon.2015.11.056

Publication date:

2016

Document Version

Peer reviewed version

Link back to DTU Orbit

Citation (APA):

Kling, J., Hansen, T. W., \& Wagner, J. B. (2016). Quantifying the growth of individual graphene layers by in situ environmental transmission electron microscopy. Carbon, 99, 261-266.

https://doi.org/10.1016/j.carbon.2015.11.056

\section{General rights}

Copyright and moral rights for the publications made accessible in the public portal are retained by the authors and/or other copyright owners and it is a condition of accessing publications that users recognise and abide by the legal requirements associated with these rights.

- Users may download and print one copy of any publication from the public portal for the purpose of private study or research.

- You may not further distribute the material or use it for any profit-making activity or commercial gain

- You may freely distribute the URL identifying the publication in the public portal

If you believe that this document breaches copyright please contact us providing details, and we will remove access to the work immediately and investigate your claim. 


\section{Quantifying the growth of individual graphene}

\section{2 layers by in situ environmental transmission}

\section{3 electron microscopy}

4 Jens Kling*, Thomas W. Hansen, Jakob B. Wagner

5 Center for Electron Nanoscopy (DTU Cen), Technical University of Denmark, Fysikvej 307, 62800 Kgs. Lyngby, Denmark

\section{Abstract}

9 The growth dynamics of layered carbon is studied by means of in situ transmission electron

10 microscopy in order to obtain a deeper insight into the growth by chemical vapor deposition,

11 which at present is the technique of choice for growing layered carbon. In situ growth of layered

12 carbon structures on nickel using acetylene as carbon precursor gas is studied in the electron

13 microscope at various gas pressures. By following the growth of individual graphene layers on

14 the Ni surface, local growth rates are determined as a function of precursor pressure. Two

15 growth regimes are identified, an initial rapid growth, which does not show a strong

16 dependence on pressure, followed by a slower growth with a strong pressure dependence.

\footnotetext{
* corresponding author: E-mail jenk@cen.dtu.dk (J. Kling), Phone: +4545256487
} 


\section{1. Introduction}

2 Carbon based materials like carbon nanotubes (CNTs) and graphene attract a lot of interest due

3 to their mechanical and electronic properties and their low mass [1]. The most efficient and

4 preferred synthesis method is chemical vapor deposition (CVD) on metallic catalysts in the

5 form of nanoparticles for CNTs [2] and polycrystalline wafers or foils for graphene. Copper

6 (Cu) is mainly used for growing single layer graphene, as it shows a self-limiting growth [3-

7 5]. One drawback of $\mathrm{Cu}$ as catalyst is the high process temperature of about $1000{ }^{\circ} \mathrm{C}$ leading

8 to technological challenges for oven material and high process energy costs. Nickel (Ni), as an

9 alternative, turns out to be a good catalyst for low-temperature graphene growth at about 450-

$10650{ }^{\circ} \mathrm{C}[6,7]$ and can grow multilayer graphene, which can be of use for certain electronic

11 applications [8] or as diffusion barrier [9] and corrosion protection [10]. However, the growth

12 process is more difficult to control especially for single or few layer graphene. The main reason

13 for the difference in controllability is assumed to be connected to the solubility of carbon in the

14 two metals, which is higher in $\mathrm{Ni}$ than in $\mathrm{Cu}$ [11]. The solidification of carbon from gaseous

carbon containing precursors to (multi-layer) graphene has been suggested to involve dissolved

carbon, which after super-saturation of the nickel leads to precipitation. The appearance of

17 carbon species at the surface and in the subsurface during growth was confirmed by X-ray

18 photoelectron spectroscopy (XPS) studies [7,12]. To control the growth of single layer graphene on the catalyst, a fundamental understanding of the growth of layered carbon itself is needed. Direct observation of the layer growth will unquestionably contribute to this. In situ transmission electron microscopy (TEM) experiments were conducted on the growth of carbon fibers on Ni nanoparticles. From these experiments, growth rates of these fibers were obtained and a growth mechanism proposed which involve the diffusion of carbon through the particle

24 [13], whereas a growth mechanism mainly involving carbon diffusion on the Ni surface was proposed elsewhere [14]. However, the dynamics of the individual layer growth was not 
1 investigated. As the situation for an extended flat surface might be completely different due to

2 the lower surface curvature and the volume of the catalyst, we perform in situ TEM on more

3 extended surfaces to get a deeper insight in the growth of individual layers and address the

4 question of growth dynamics.

\section{$5 \quad$ 2. Experimental}

6 In order to mimic the flat surface of Ni foils or wafers used in large-scale ex situ CVD growth

7 of layered carbon, but are unsuited for TEM investigations, nickel oxide (NiO) powder with a

8 particle size up to a few hundred nanometers was used as a precursor for the catalyst in the

9 present study. Furthermore, the geometry of the NiO particles provides extended Ni surfaces

10 with small curvature after reduction, allowing for direct observation of the (multi-layered)

11 graphene in the environmental transmission electron microscope (ETEM). The small curvature

12 is clearly different to the large curvature of nanometer sized Ni particles which result in carbon

13 nanotube growth [14]. $\mathrm{NiO}$ resembles the native oxidized surface of $\mathrm{Ni}$, which is always

14 present when stored under ambient conditions requiring a reduction procedure before CVD

15 growth.

16 In situ electron microscopy growth of layered carbon structures and corresponding analysis

17 using an ETEM is performed by exposing $\mathrm{Ni}$ to a precursor gas atmosphere of acetylene $\left(\mathrm{C}_{2} \mathrm{H}_{2}\right)$.

18 The ETEM is operated at $300 \mathrm{kV}$ in order to optimize the microscope performance in terms of

19 resolution and signal-to-noise ratio while operating in a gaseous atmosphere [15], even though

20 this is above the threshold for knock-on damage of carbon atoms within a graphene sheet [16].

21 Great care was taken to minimize electron beam damage of the grown carbon structures. Under

22 the chosen conditions, with the dose rate at a maximum of about $2 \times 10^{6} \mathrm{e}^{-/}\left(\mathrm{nm}^{2} \mathrm{~s}\right)$, no obvious

23 degradation of the grown graphene layers was observed. The electron microscope itself is 
1 cleaned by bake-out of the gas feed lines and internal plasma cleaning between experiments in

2 order to minimize cross contamination between individual experiments.

3 The NiO powder is dispersed on a micro electro-mechanical systems (MEMS)-based heating 4 chip with a holey $\mathrm{Si}_{3} \mathrm{~N}_{4}$ membrane on top (DENSsolutions). The samples are plasma cleaned 5 ( $\mathrm{Ar} / \mathrm{O}_{2}$ gas) prior to insertion in the ETEM in order to reduce surface contamination. Moisture 6 within the porous structure of the $\mathrm{NiO}$ agglomerates is minimized by heating the sample to 350

$7{ }^{\circ} \mathrm{C}$ for about $30 \mathrm{~min}$ in the microscope. The $\mathrm{NiO}$ sample is then reduced in situ in the ETEM at $8500{ }^{\circ} \mathrm{C}$ in flowing hydrogen $\left(\mathrm{H}_{2}\right)$ building up to a pressure of $1.2 \mathrm{mbar}$ for $1 \mathrm{~h}$. The temperature 9 is increased to the growth temperature of $650{ }^{\circ} \mathrm{C}$ and $\mathrm{C}_{2} \mathrm{H}_{2}$ gas is added to the $\mathrm{H}_{2}$ gas flow. 10 This procedure ensures that the catalyst is maintained in a consistent and reproducible state of 11 metallic Ni and prevents high-temperature reoxidation due to residual oxidizing agents in the 12 ETEM. With the proposed procedure a clean system is ensured, leading to a reproducible and 13 successful route to graphene growth. 


\section{3. Results}

2 To confirm that $\mathrm{NiO}$ is reduced to catalytic active metallic $\mathrm{Ni}$ in the electron microscope,

3 spectroscopic information by means of electron energy-loss spectroscopy (EELS) are acquired

4 during reduction in $\mathrm{H}_{2}$. The spectra acquired from extended surface areas of individual catalyst

5 particles (up to at least $20 \mathrm{~nm}$ from the particle surface) clearly show the reduction from $\mathrm{NiO}$

6 to metallic Ni (Fig. 1). The fine-structure of the Ni L2/3-edge observed at an energy-loss
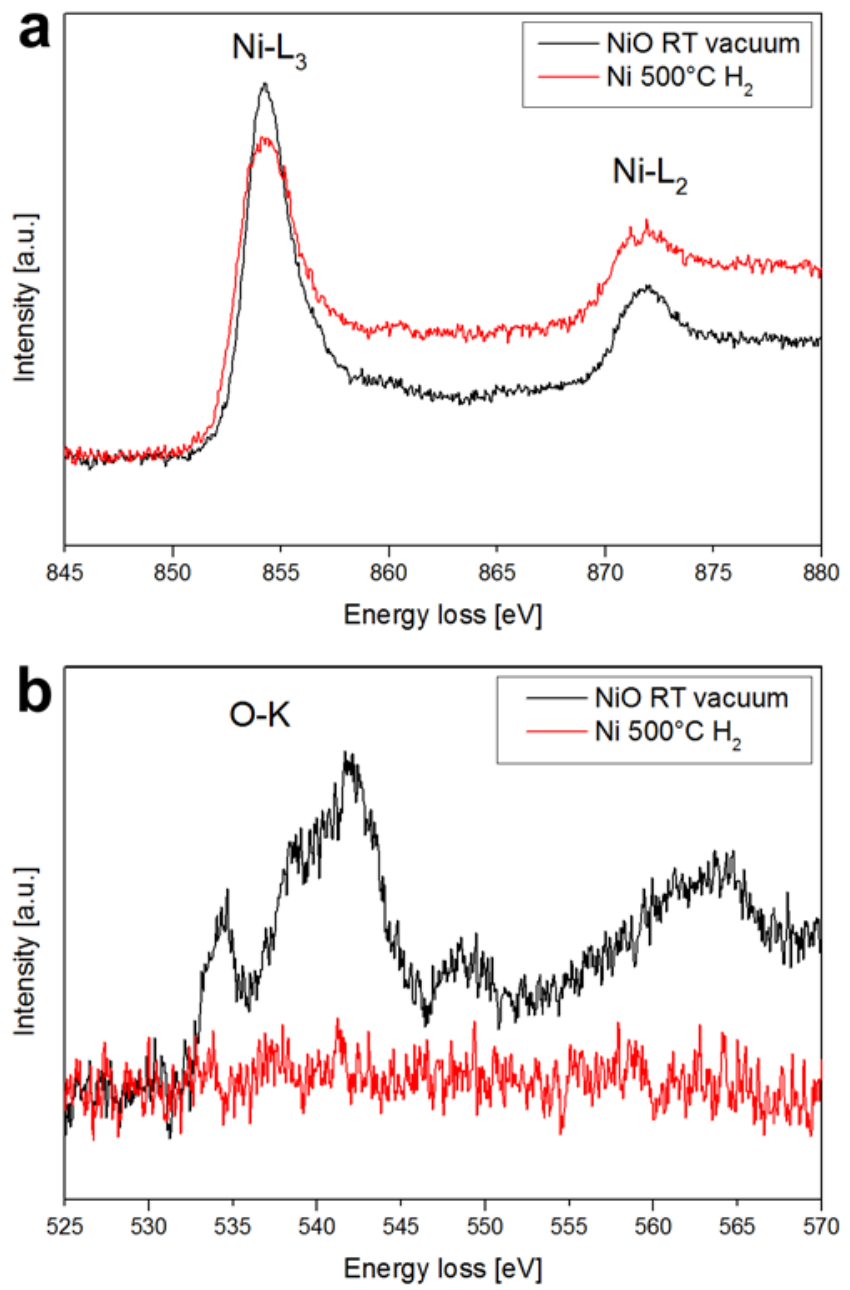

Figure 1: Electron Energy-Loss Spectra of the catalyst in vacuum (black) and under $\mathrm{H}_{2}$-atmosphere after reduction (red) in the electron microscope. The change in the fine structure of the $\mathrm{Ni} \mathrm{L}_{2 / 3}$-edge (a) combined with the absence of the oxygen $\mathrm{K}$-edge after reduction (b) indicates the success of reducing the catalyst from $\mathrm{NiO}$ to $\mathrm{Ni}$. 
1 between approx. $855 \mathrm{eV}$ and $880 \mathrm{eV}$ in the black and red spectra in Fig. 1a is characteristic of

2 oxidized and metallic nickel, respectively [17]. Additionally, the O K-edge at about $532 \mathrm{eV}$

3 (Fig. 1b) is absent in the reduced state. Therefore, a full reduction of the surface area of at least

$420 \mathrm{~nm}$ thickness is confirmed. Possible unreduced $\mathrm{NiO}$ fractions within the Ni particles or

5 agglomerates, as reported previously [18], are neglected and no influence of reduction products

6 on the environment is assumed. In order to check for carbon contaminants on the catalyst before

7 initiating the growth, the EELS signal around $284 \mathrm{eV}$ is also acquired (carbon K-edge). No

8 sign of carbon was observed, which means that if carbon is present, it is below the detection

9 limit.

10 To investigate the pressure dependence of the growth of carbon layers, two different partial

11 pressures of $\mathrm{C}_{2} \mathrm{H}_{2}$ of approximately $1 \times 10^{-4}$ and $3 \times 10^{-4}$ mbar were used. Figure 2 shows the

12 development from single/few layer graphene to a relatively thick graphitic structure illustrated

13 by stills from acquired movies of the dynamic process. The in situ growth results in well-

14 aligned carbon layers with an inter-layer distance of about $0.34 \mathrm{~nm}$ corresponding to the

15 interlayer distance in graphite. Furthermore, EELS of the grown layered material shows a

16 strong carbon signal (Fig. 2d) characteristic of layered graphitic carbon. 
1 Quantitative data from the in situ growth is extracted by plotting the number of carbon layers

2 formed as function of time. Only fully-formed carbon layers (within the field of view) are

3 considered in the growth analysis. Figure 3 shows the layer growth rate extracted from

4 observation of growth at a $\mathrm{C}_{2} \mathrm{H}_{2}$ pressure of approx. $3 \times 10^{-4} \mathrm{mbar}$. The data is extracted from

5 three distinct areas and shows the same trend. The data from area 1 and 2 are aligned in time
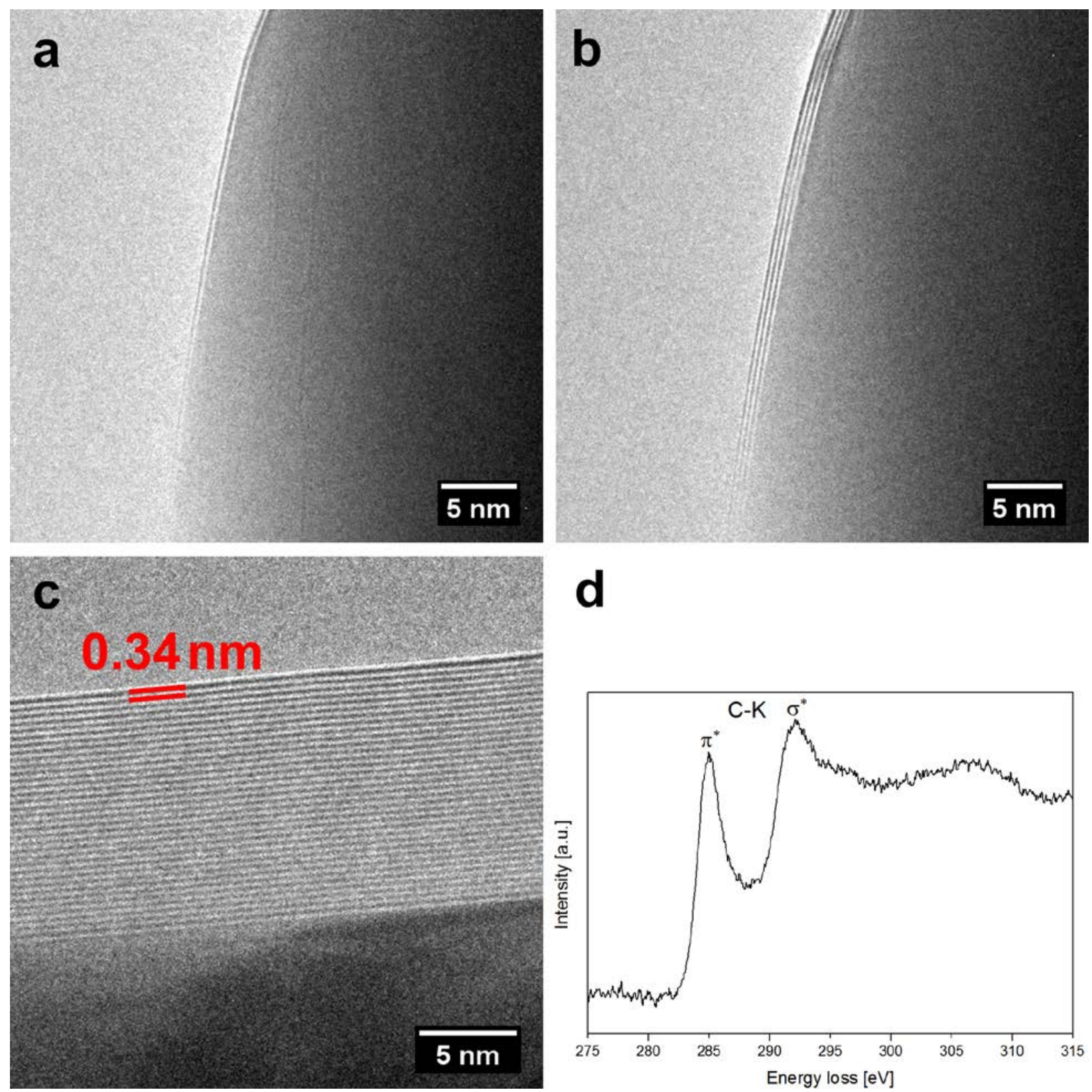

Figure 2: Images from the experiments at growth $\left(650^{\circ} \mathrm{C}, \mathrm{P}\left(\mathrm{C}_{2} \mathrm{H}_{2}\right)=3 \times 10^{-4} \mathrm{mbar}\right)$. The layered structure on the Ni surface is clearly visible. EELS data from the layered structure clearly shows the carbon K-edge with the characteristic $\pi^{*}$ and $\sigma^{*}$ bands. 
1 at the observation of 4 formed layers, the data of area 3 is aligned to 2 for 42 formed layers.

2 Missing data in the growth curves are either due to frames, which could not be analyzed as the

3 image was out of focus, severe drift or not acquired due to limited data file buffer size.

4 The measured data are fitted linearly in six time regimes (a-f), which give the layer growth rate

5 as a function of time / carbon structure thickness. Figure 3 shows at least two different growth

6 regimes. After an initial high layer growth rate (a and b), the growth slows down in the range

7 of 10 to 15 carbon layers (c and d), and slows down further after 30-40 layers are formed (e

8 and $\mathrm{f})$. The initial growth is relatively fast with a rate of $0.6-0.8$ layer/s. As the number of layers

9 increase the observed growth rate decreases to 0.19 layer/s in the timeframe of the experiment.

10 The pressure dependence of the growth in general and the growth rate in particular is studied

11 by repeating the experiment with a $\mathrm{C}_{2} \mathrm{H}_{2}$ pressure of $1 \times 10^{-4} \mathrm{mbar}$. The decreased precursor

12 pressure still result in aligned carbon layers on top of the Ni catalyst. Again, two regimes are

13 found in the layer growth rate as shown in Fig. 4. The first 30 layers grow rapidly, with a

14 growth rate of about 0.4 layer/s. As in the 'high-pressure' case, for higher number of layers

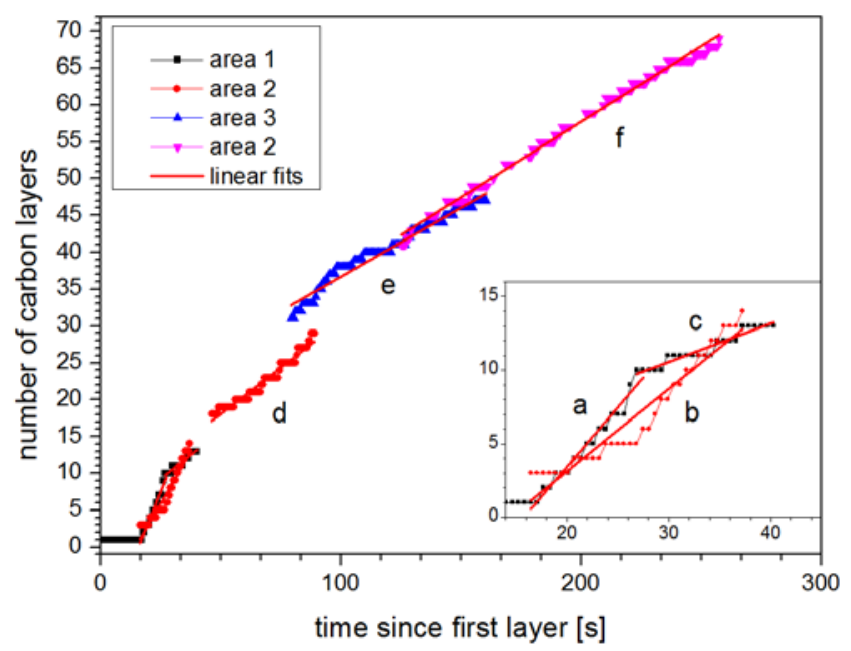

Figure 3: Number of carbon layers per time extracted from in situ TEM movies of the growth at three distinct areas. a-f give the distinct linear fits. $T=650{ }^{\circ} \mathrm{C}, P\left(\mathrm{C}_{2} \mathrm{H}_{2}\right)=3 \times 10^{-4}$ mbar. 
1 (here about 30) the growth rate decreases significantly to 0.02 layer/s. Apparently, the initial

2 growth rate is only slightly lower for the low pressure $\left(1 \times 10^{-4} \mathrm{mbar}\right)$ study compared to the

3 high pressure $\left(3 \times 10^{-4}\right.$ mbar) indicating that the underlying mechanism at this stage is less

4 pressure-dependent. However, the growth rate after approx. 30 layers is an order of magnitude

5 lower for the low pressure study than for the high pressure study. The in situ measured growth

$6 \quad$ rates are summarized in Tables 1 and 2.

$7 \quad$ Table 1: Layer by layer growth rates extracted from linear fits of in situ observed layer growth (Fig. 3).

$8 T=650^{\circ} \mathrm{C}, \mathrm{P}\left(\mathrm{C}_{2} \mathrm{H}_{2}\right)=3 \times 10^{-4} \mathrm{mbar}$.

layer/s standard error

\begin{tabular}{lll}
\hline a & 0.81 & 0.04 \\
b & 0.56 & 0.03 \\
c & 0.26 & 0.02 \\
d & 0.25 & 0.01 \\
e & 0.19 & 0.01 \\
f & 0.21 & 0.01 \\
\hline
\end{tabular}

9

10 Table 2: Layer by layer growth rates extracted from linear fits of in situ observed layer growth (Fig. 4).

$11 \mathrm{~T}=650^{\circ} \mathrm{C}, \mathrm{P}\left(\mathrm{C}_{2} \mathrm{H}_{2}\right)=1 \times 10^{-4} \mathrm{mbar}$

\begin{tabular}{rrr} 
& layer/s & standard error \\
\hline a & 0.43 & 0.01 \\
b & 0.02 & 0.01 \\
\hline
\end{tabular}

12 
1 In order to study the growth in greater detail, the in-plane layer growth rate, the growth rate of

2 projected individual graphene layers is extracted from the acquired movies. The frames are

3 spatially aligned using the plugin StackReg in Image [19]. After the first graphene layer

4 formed at the Ni surface, additional carbon layers are formed at the nickel/carbon interface by

5 a ledge growth mechanism as illustrated in Fig. 5. The relative position of the growth front

6 (illustrated by arrows) is extracted from individual frames and converted to a growth rate. In

7 some cases two layers are observed to grow simultaneously within the same limited field of

8 view. The projected rate of the in-plane layer growth is plotted for several layers in Fig. 6 and

97 (the color coding is identical to Fig. 3 and 4). In both experiments the growth rate appears

10 very scattered, but is clearly slower in the lower pressure range, which matches the decreased

11 layer growth rate. However, in the higher pressure range there seems to be a trend of the in-

12 plane growth rate slowing down with time. This becomes apparent when comparing the data

13 points of growth regimes $d$ and $\mathrm{f}$. These data come from the same area at different growth

14 times. Correlating the layer growth rate with the in-plane growth rate, both seem to be

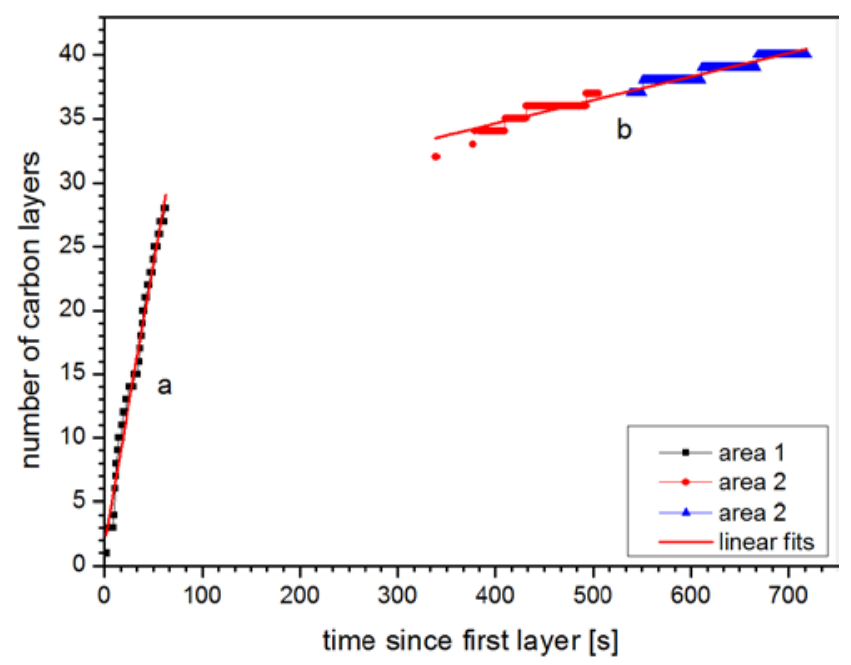

Figure 4: Number of carbon layers per time extracted from in situ TEM movies of the growth at two distinct areas. $a$ and $b$ give the distinct linear fits. $T=650{ }^{\circ} \mathrm{C}, P\left(\mathrm{C}_{2} \mathrm{H}_{2}\right)=1 \times 10^{-4} \mathrm{mbar}$. 
1 connected, as for the higher layer growth rate ( $\mathrm{d}$ compared to $\mathrm{f}$ in Fig. 3) the in-plane growth

2 rate also seem to have a tendency of being higher (red and pink data points of area 2 in Fig. 6).
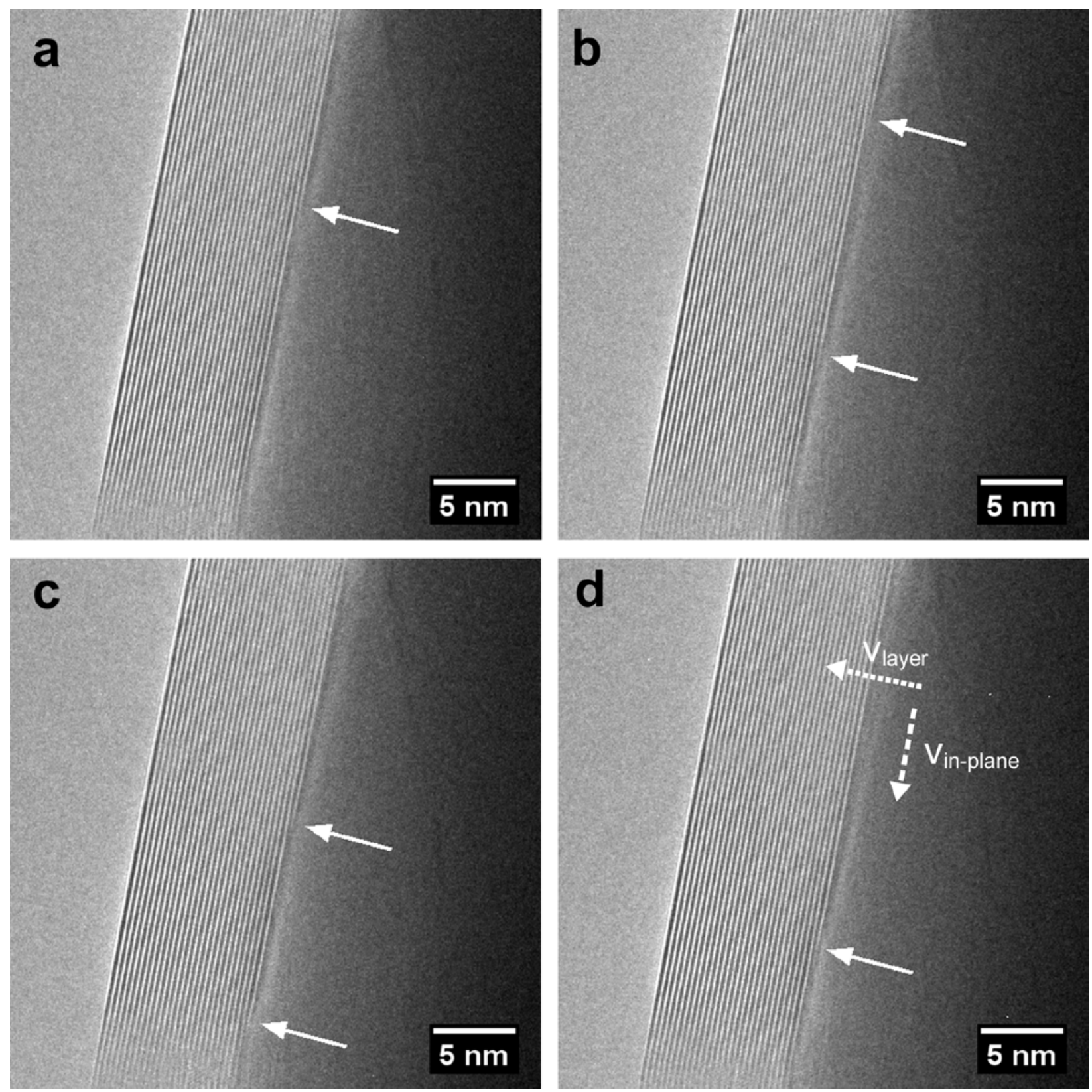

Figure 5: Image series with $0.61 \mathrm{~s}$ between the images. Following the arrow, one can follow the in-plane growth of the carbon layers. $(d)$ dashed arrow ( $\left.v_{\text {in-plane }}\right)$ indicates the in-plane growth, dotted arrow ( $\left.v_{\text {layer }}\right)$ indicates the layer growth. $T=650^{\circ} \mathrm{C}, P\left(\mathrm{C}_{2} \mathrm{H}_{2}\right)=3 \times 10^{-4} \mathrm{mbar}$. 


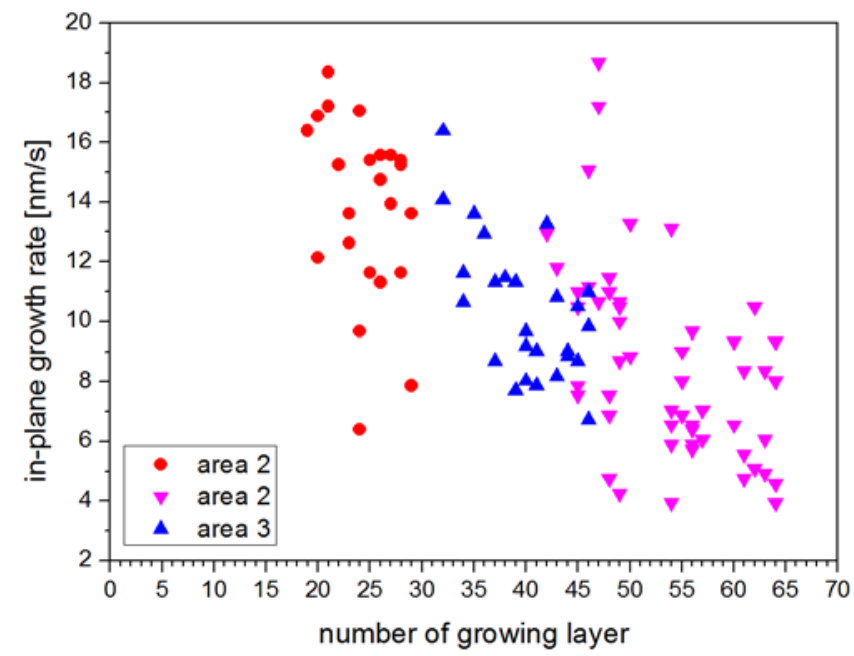

Figure 6: Growth rate in-plane; growth rate for growth regime $d$, e and $f$ (Fig. 3). $T=650^{\circ} \mathrm{C}, P\left(\mathrm{C}_{2} \mathrm{H}_{2}\right)=3 \times 10^{-4}$ mbar. Colors correspond to colors in Figure 3.

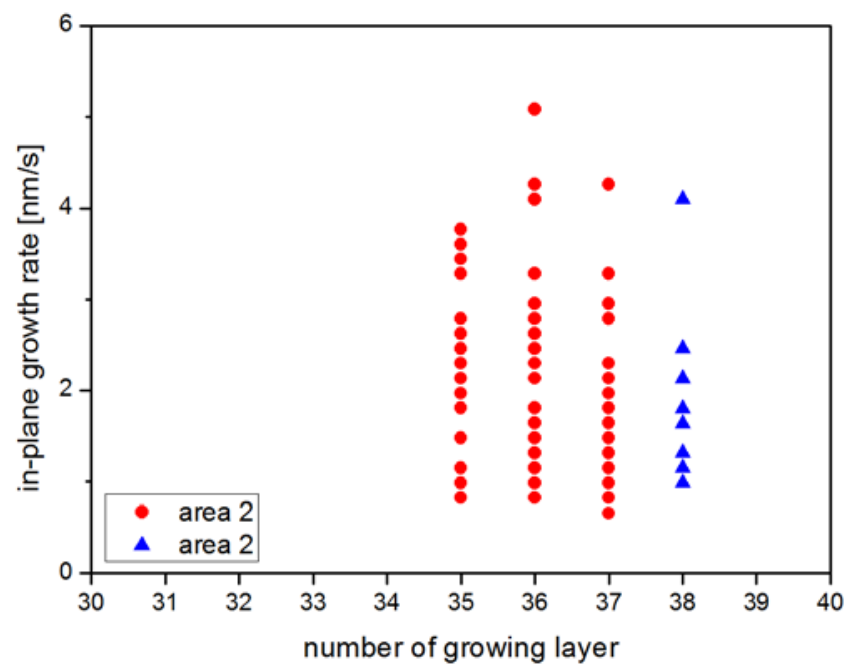

Figure 7: Growth rate in-plane; growth rate for growth regime $b$ (Fig. 4). $T=650^{\circ} \mathrm{C}, \mathrm{P}\left(\mathrm{C}_{2} \mathrm{H}_{2}\right)=1 \times 10^{-4}$ mbar. Colors correspond to colors in Figure 4.

\section{4. Discussion}

2 The fast initial growth might be connected to the solubility of carbon in Ni, as found in XPS

3 studies [7,12,20]. After carbon (super)saturation of the first few atomic Ni layers, a graphene

4 seed nucleates and carbon diffuse to the surface and nucleate at the seed resulting in the

5 formation of the first graphene layer. This is in agreement with the model suggested by Patera

6 et al. [20]. If carbon for the initial growth is coming from the Ni bulk, the growth rate could be

7 assumed to be less precursor pressure dependent as it is limited by the diffusion kinetics in 
1 solid nickel. However, the absolute time for saturation is dependent on the gas pressure, which

2 influence the incubation time. Furthermore, the carbon depth profile is influenced by kinetics

3 and might show pressure dependence. With higher pressure more carbon can diffuse into Ni at

4 once. But as carbon has a very limited solubility in Ni, the first $\mathrm{Ni}$ sublayers are saturated faster

5 meaning the thickness of saturated Ni sublattice is thin, the depth profile is shallow. At lower

6 pressure less carbon diffuse into $\mathrm{Ni}$ at once and it takes longer to saturate the first Ni sublayers.

7 This means carbon can diffuse deeper into the Ni sublattice until saturation of the first layers

8 is reached, the depth profile should reach deeper. So the thickness of Ni sublattice with

9 dissolved carbon is thicker for lower pressures (more carbon dissolved), which leads to more

10 layer growth in the first growth regime. This matches the observation of up to about 30 layers

11 in the faster growth regime for the lower pressure and about 15 for the higher pressure.

12 Nevertheless, from the Ni-C phase diagram [21] a maximum solubility of carbon under 13 equilibrium conditions of about 2.7 at $\%$ is reported, which can be extended up to about 7.4 at\% at $1314{ }^{\circ} \mathrm{C}$ in a metastable phase. By calculating the volume and surface of a Ni sphere $100 \mathrm{~nm}$ in diameter and the number of carbon atoms in a single graphene layer covering this surface, the carbon concentration needed is about 2.4 at\% close to the saturation concentration, assuming a homogeneous distribution of carbon in the Ni sphere. For 10 layers a concentration of about 20 at\% is needed. This rough estimate already shows that the carbon for the numerous graphene layers cannot solely come from the dissolved carbon in the $\mathrm{Ni}$.

The slower and apparently more pressure-dependent growth regime fits a diffusion driven mechanism, namely the carbon atoms to the active Ni surface at the Ni-graphene interface from the gas phase itself. The energy barrier for diffusion of carbon atoms at the Ni-graphene interface is low (about $0.5 \mathrm{eV}$ ) and additional carbon atoms tend to nucleate at the existing graphene edge $[14,22]$. Step edges which should facilitate the growth of graphene cannot be observed clearly, which might be due to the projected image of the rather large particle. 
1 The growth of numerous graphene layers in the experiments has two prerequisites: access of

2 the gaseous specie to the Ni surface to decompose into carbon atoms, and diffusion of the

3 carbon atoms to the growth front. Diffusion through the defect free graphene layers is unlikely

$4 \quad[23,24]$, but as we are dealing with grown graphene layers on rather large particles and particle

5 agglomerates, the presence of defects in the graphene layers, can allow gas to diffuse to the C-

6 Ni interface, or areas with little or no graphene layers, can act as gateways for carbon precursor

7 to the catalytic surface. It has been reported that carbon can easily diffuse at the Ni-graphene

8 interface and the Ni subsurface [14,22], but the diffusion length in our experiment still remains

9 long. From the growth movies of the present experiments the carbon diffusion at the Ni

10 (sub)surface has to be at least several tens of nanometers from source to growth front as no

11 defects in the layered carbon was observed in the field of view. This rather long diffusion length

12 and the small curvature geometry of the catalyst particles shows the clear difference to carbon

13 nanotube growth and the underlying mechanisms, as the particles size for nanotube growth

14 ranges below $10 \mathrm{~nm}$. The results obtained here should be much closer to the flat wafer or foil

15 geometry of a large scale CVD process.

16 The scatter of the in-plane growth rate found in both, the 'high pressure' experiment and the

17 'low pressure' experiment indicates that the formation of carbon layers might be difficult to

18 control. Especially the scattering within the growth of one layer in the lower pressure experiments suggests a strong dependency of the substrate structure, like orientation and defects [25-27], which is subject to future in situ studies. However, in the present work we were not able to uniquely determine the surface termination of the $\mathrm{Ni}$ during growth.

22 Furthermore, several layers of carbon are formed very quickly on the nickel surface in the 23 initial stage. This process seems to be less pressure dependent than the latter growth process, making it very hard to limit the growth to single or double layer graphene under the present growth conditions. 
1 As the growth rate is extracted from a projection, and influenced by the actual number of carbon

2 atoms joining the carbon layers per time depending on the thickness of the sample

3 perpendicular to the electron beam needs to be taken into account. For the same particle, this

4 effect should be negligible as the grain thickness should not change within the recorded movie.

\section{5. Conclusion}

6 Successful in situ graphene growth on $\mathrm{Ni}$ at $650{ }^{\circ} \mathrm{C}$ in the ETEM was performed. From movies

7 recorded during growth, layer growth rates and in-plane growth rates were extracted. At least

8 two growth regimes were observed: an initial fast regime for the first few layers followed by a

9 slower regime for higher layer numbers. The in-plane growth rate appears to correlate to the

10 layer growth rate. Furthermore, the slower growth regime shows pressure dependence, whereas

11 the faster growth regime seems to be significantly less pressure dependent. A resulting

12 thickness of the perfectly aligned carbon structures of tens of nanometers indicate a continuous

13 supply of carbon to the growth front from the gas phase. 


\section{Supplementary}

2 Three growth movies are included as supporting information. Movie1.avi showing the initial

3 growth at higher pressure, Movie2.avi showing the slower growth after few layers growth at

4 higher pressures, Movie3.avi showing the slower growth after few layers growth at lower

5 pressures. This material is available online.

6

7 Acknowledgment

8 This work was funded by the 7th Framework project "GRAFOL". The A.P. Møller and

9 Chastine Mc-Kinney Møller Foundation is acknowledged for their contribution toward the

10 establishment of the Center for Electron Nanoscopy in the Technical University of Denmark.

11 Thanks to Søren B. Simonsen and Quentin Jeangros for providing the NiO samples.

\section{References}

[1] Ferrari AC. Science and technology roadmap for graphene, related two-dimensional crystals, and hybrid systems. Nanoscale 2014;online.

[2] Tessonnier J-P, Su DS. Recent progress on the growth mechanism of carbon nanotubes: a review. ChemSusChem 2011;4:824-47.

[3] Mattevi C, Kim H, Chhowalla M. A review of chemical vapour deposition of graphene on copper. J Mater Chem 2011;21:3324.

[4] Lee Y, Bae S, Jang H, Jang S, Zhu S-E, Sim SH, et al. Wafer-Scale Synthesis and Transfer of graphene films. Nano Lett 2010;10:490-3.

[5] Li X, Cai W, An J, Kim S, Nah J, Yang D, et al. Large-area synthesis of high-quality and uniform graphene films on copper foils. Science 2009;324:1312-4.

[6] Weatherup RS, Amara H, Blume R, Dlubak B, Bayer BC, Diarra M, et al. Interdependency of Subsurface Carbon Distribution and Graphene - Catalyst 
[7] Weatherup RS, Bayer BC, Blume R, Ducati C, Baehtz C, Schlögl R, et al. In situ characterization of alloy catalysts for low-temperature graphene growth. Nano Lett 2011;11:4154-60.

[8] Han T-H, Lee Y, Choi M-R, Woo S-H, Bae S-H, Hong BH, et al. Extremely efficient flexible organic light-emitting diodes with modified graphene anode. Nat Photonics 2012;6:105-10.

[9] Hong J, Lee S, Lee S, Han H, Mahata C, Yeon H-W, et al. Graphene as an atomically thin barrier to Cu diffusion into Si. Nanoscale 2014;6:7503-11.

[10] Prasai D, Tuberquia JC, Harl RR, Jennings GK, Bolotin KI. Graphene: corrosioninhibiting coating. ACS Nano 2012;6:1102-8.

[11] Seah C-M, Chai S-P, Mohamed AR. Mechanisms of graphene growth by chemical vapour deposition on transition metals. Carbon N Y 2014;70:1-21.

[12] Weatherup RS, Bayer BC, Blume R, Baehtz C, Kidambi PR, Fouquet M, et al. On the mechanisms of Ni-catalysed graphene chemical vapour deposition. Chemphyschem 2012;13:2544-9.

[13] Baker RTK, Barber MA, Harris PS, Feates FS, Waite RJ. Nucleation and growth of carbon deposits from the nickel catalyzed decomposition of acetylene. J Catal 1972;26:51-62.

[14] Helveg S, Lopez-Cartes C, Sehested J, Hansen PL, Clausen BS, Rostrup-Nielsen JR, et al. Atomic-scale imaging of carbon nanofibre growth. Nature 2004;427:426-9.

[15] Wagner JB, Cavalca F, Damsgaard CD, Duchstein LDL, Hansen TW. Exploring the environmental transmission electron microscope. Micron 2012;43:1169-75.

[16] Zobelli A, Gloter A, Ewels C, Seifert G, Colliex C. Electron knock-on cross section of carbon and boron nitride nanotubes. Phys Rev B 2007;75:245402.

[17] Leapman R, Grunes L, Fejes P. Study of the L 23 edges in the $3 \mathrm{~d}$ transition metals and their oxides by electron-energy-loss spectroscopy with comparisons to theory. Phys Rev B 1982;26:614.

[18] Jeangros Q, Hansen TW, Wagner JB, Damsgaard CD, Dunin-Borkowski RE, Hébert C, et al. Reduction of nickel oxide particles by hydrogen studied in an environmental TEM. 
J Mater Sci 2012;48:2893-907.

[19] Thévenaz P, Ruttimann UE, Unser M. A pyramid approach to subpixel registration based on intensity. IEEE Trans Image Process 1998;7:27-41.

[20] Patera LL, Africh C, Weatherup RS, Blume R, Bhardwaj S, Castellarin-Cudia C, et al. In Situ Observations of the Atomistic Mechanisms of Ni Catalyzed Low Temperature Graphene Growth. ACS Nano 2013:7901-12.

[21] Singleton M, Nash P. The C-Ni (Carbon-Nickel) system. Bull Alloy Phase Diagrams 1989;10:121-6.

[22] Abild-Pedersen F, Nørskov JK, Rostrup-Nielsen JR, Sehested J, Helveg S. Mechanisms for catalytic carbon nanofiber growth studied by ab initio density functional theory calculations. Phys Rev B - Condens Matter Mater Phys 2006;73:1-13.

[23] Bunch JS, Verbridge SS, Alden JS, Van Der Zande AM, Parpia JM, Craighead HG, et al. Impermeable atomic membranes from graphene sheets. Nano Lett 2008;8:2458-62.

[24] Leenaerts O, Partoens B, Peeters FM. Graphene: A perfect nanoballoon. Appl Phys Lett 2008;93:1-4.

[25] Abild-Pedersen F, Nørskov JK, Rostrup-Nielsen JR, Sehested J, Helveg S. Mechanisms for catalytic carbon nanofiber growth studied by ab initio density functional theory calculations. Phys Rev B - Condens Matter Mater Phys 2006;73:1-13.

[26] Saadi S, Abild-Pedersen F, Helveg S, Sehested J, Hinnemann B, Appel CC, et al. On the role of metal step-edges in graphene growth. J Phys Chem C 2010;114:11221-7.

[27] Takahashi K, Yamada K, Kato H, Hibino H, Homma Y. In situ scanning electron microscopy of graphene growth on polycrystalline Ni substrate. Surf Sci 2012;606:72832. 